Artículo

\title{
Un paso al frente: el sector metalmecánico asturiano ante la reconversión industrial, 1978-2000
}

\section{Guillermo Antuña* $\mathbb{D}$ \\ Universidad de Barcelona}

\section{INFORMACIÓN DEL ARTÍCULO}

\section{Historia del artículo:}

Recibido: 11 de mayo de 2020

Aceptado: 8 de enero de 2021

On-line: 15 de marzo de 2021

\section{Códigos JEL}

L61

N64

N94

F6

Palabras clave

Metalmecánico

Industria

Reconversión

Asturias

\section{JEL classification:}

L61

N64

N94

F6

Keywords:

Metal-mechanic

Industry

Restructuring

Asturias

\section{R E S U M E N}

Este trabajo analiza la evolución del tejido metalmecánico asturiano entre la reconversión industrial y el cambio de milenio, y matiza la visión de Asturias como un territorio desindustrializado. Desde mediados del siglo XIX, la aglomeración de actividades transformadoras en torno al carbón y la metalurgia favoreció la generación progresiva de economías externas. Estas terminarían por generar un entorno clusterizado, que permitió al metalmecánico superar el declive de los grandes sectores hegemónicos y reorientar su actividad más allá de sus mercados tradicionales. Dicho salto estuvo liderado por una base empresarial emergente, de clara vocación internacional y especializada en proyectos de alto componente tecnológico. A su vez, este enfoque podría suponer una nueva línea de investigación en el estudio de otras regiones siderúrgicas en declive mediante el análisis de dinámicas de largo plazo que desemboquen en el desarrollo de actividades relacionadas con la industria 4.0.

@ 2021 Asociación Española de Historia Económica

\section{A step forward: metal-mechanic sector in Asturias facing the industrial restructuring, 1978-2000}

\section{A B S T R A C T}

This paper analyzes the evolution of the Asturian metal-mechanic network between the industrial restructuring and the turn of the millennium, showing a revisited view of Asturias as a deindustrialized territory. The agglomeration of processing activities around mining and metallurgy since the mid-19th century triggered the progressive generation of external economies, which ended up generating a clustered environment that would allow the metal-mechanic industry to overcome the downturn of the big hegemonic sectors and to reorient its activity beyond its traditional markets. The leap was taken by an emerging set of internationally focused companies, specialized in high-tech projects. Moreover, this approach could represent a new line of research in the study of other steel-producing regions in decline, through the analysis of long-term dynamics that lead to the development of activities related to Industry 4.0.

@ 2021 Asociación Española de Historia Económica

\footnotetext{
* Autor para correspondencia. Correo electrónico: g.antuna@ub.edu (Guillermo Antuña Martínez). 


\section{Introducción}

El sector metalmecánico ha sido históricamente considerado como eje vertebrador y motor del desarrollo industrial por su función de catalizador tecnológico para el resto de actividades fabriles. El incremento sostenido de la productividad manufacturera se ha basado en dicho avance técnico, que ha permitido mantener la competitividad de las empresas y, con ellas, de las economías industriales (Landes, 1969; Pollard, 1981; Sanchís y Cubel, 2007). Pese a todo, la historia económica no ha prestado demasiada atención a la evolución de este sector, y ha tendido a centrarse bien en alguno de los grandes subsectores que lo integran y que por su propio peso han merecido estudios específicos, caso del automóvil o de la construcción naval ${ }^{1}$, bien a utilizarlo como elemento transversal para el análisis de otras realidades, caso del papel de la importación de maquinaria y componentes en el desarrollo económico de un territorio (Catalan, 1995; Eaton y Kortum, 2001; Ducoing y Tafunell, 2013).

El metalmecánico es un sector aún por definir, una suerte de cajón de sastre conformado por diferentes ramas de actividad aparentemente muy dispares entre sí. Por este motivo resulta impreciso referirse a él como un conjunto unitario a nivel nacional, ya que tanto su estructura como su devenir histórico varían profundamente de un territorio a otro, en función de la especialización industrial de la propia región en que se ubique.

En este sentido, Asturias es un claro ejemplo de región cuyo desarrollo industrial responde históricamente a un modelo basado en el aprovechamiento de ventajas comparativas derivadas de su dotación de recursos naturales. Desde mediados del siglo XIx el carbón determinaría su estructura económica, así como su alto grado de especialización en el sector metalúrgico, factor clave para comprender la trayectoria del principado en el largo plazo (Martínez-Galarraga, Rosés y Tirado, 2015; Díez-Minguela, Martínez-Galarraga y Tirado, 2018)2 ${ }^{2}$. La hulla de los valles del Nalón y el Caudal sería también el principal reclamo para la implantación de ENSIDESA (Empresa Nacional Siderúrgica Sociedad Anónima) en los primeros años cincuenta. Desde entonces, el metalmecánico mantendría con la industria matriz una relación bidireccional y endogámica, confluyendo así los factores de oferta y demanda operantes en la localización industrial. Ante la falta de una estructura industrial diversificada, en Asturias la siderurgia ejercía como proveedor de acero mientras, simultáneamente, se erigía como cliente principal para los productos metálicos y, en menor medida, para la maquinaria ${ }^{3}$.

Por tanto, sería lógico pensar que la crisis y posterior reconversión siderúrgica acarrearían el declive metalmecánico en la región. Las investigaciones en historia económica relativas a la reconversión industrial la han estudiado como un proceso homogéneo, centrándose exclusivamente en los grandes sectores industriales en declive. Por este mismo motivo, aún perdura la idea de que Asturias quedó reducida a un páramo industrial desde los años ochenta, aunque los datos no respaldan esta creencia: en el año 2018, el sector metalmecánico ocupaba en Asturias a 18878 personas, un 36\% del empleo industrial

\footnotetext{
1 Como ejemplo, véase Catalan (2017) y Valdaliso (2003), respectivamente. 2 Ambas obras permiten seguir, en función del PIB, la posición relativa de Asturias respecto al resto de regiones españolas desde 1860.

3 Relación ya identificada en el informe La industria siderometalúrgica en Asturias (1971). SADEI (Sociedad Asturiana de Estudios Económicos e Industriales) y NEI (Nederlands Economisch Instituut).
}

de la región y un $5 \%$ del total. A su vez, el conjunto representaba un $27 \%$ del VAB industrial y un $26 \%$ de sus exportaciones manufactureras, por valor de 1144 millones de euros ${ }^{4}$.

Esta posición parece haberse logrado mediante el aprovechamiento de las externalidades propias de un entorno clusterizado, fraguado durante décadas gracias a las capacidades generadas por la aglomeración metalmecánica en torno a la metalurgia básica. El interés sobre este tipo de dinámicas surge a finales del siglo xIx, cuando en sus estudios sobre organización industrial Alfred Marshall identificó tres tipos de ventajas competitivas -economías externas- derivadas de la concentración geográfica de la actividad empresarial: la existencia de una oferta estable de mano de obra especializada, de una red de proveedores de bienes intermedios y empresas auxiliares, y de un entorno que favorezca la propagación del conocimiento, la innovación y el desarrollo técnico (Marshall, 1963). Dichas externalidades articulan el conocido como distrito marshalliano (Catalan, Miranda y Ramon-Muñoz, 2011).

Posteriormente, Michael Porter volverá sobre la tríada marshalliana para formular su teoría sobre clusters y competitividad. Así, partiendo de las externalidades antes mencionadas, Porter define un cluster como la concentración geográfica de empresas, proveedores y agentes transversales, relacionados a través de externalidades de distinta naturaleza (Porter, 1998; Porter y Ketels, 2009). A su vez, estas entidades han revitalizado el papel de las pequeñas y medianas empresas en los mercados globales, al permitirles superar sus límites de capacidad incrementado su productividad y generando un entorno favorable a la internacionalización (Valdaliso, 2010).

Con este trabajo se aspira a abrir una nueva línea de investigación sobre un sector muy poco tratado, tomando como referencia una de las regiones que más sufriría el proceso de reconversión para, además, ofrecer una visión alternativa de este último: la de las industrias que crecieron en torno a los gigantes y consiguieron sobrevivir más allá de su ocaso. El presente artículo trata de reconstruir la andadura del sector metalmecánico entre el inicio de la reconversión industrial y el cambio de milenio, así como de analizar los factores que propiciaron su desarrollo. Como hipótesis de partida se sostiene que el sector aprovechó las capacidades generadas por su aglomeración en torno a la siderurgia para reinventarse y superar el declive de esta última a través de un proceso de clusterización que le permitiría dar el salto a los mercados internacionales.

El trabajo se inicia con una breve nota metodológica. El apartado 3 describe el cambio de modelo que desembocaría en la reconversión industrial. El cuarto apartado recoge la evolución del sector metalmecánico asturiano entre los años 1978 y 2000. En el quinto se pretende demostrar la existencia de un cluster metalmecánico asturiano. Por último, en el apartado 6 se detallan las conclusiones y se proponen futuras líneas de investigación.

\section{El tejido industrial metalmecánico como objeto de estudio}

La conceptualización realizada sobre el sector metalmecánico en este trabajo se lleva a cabo desde la visión de las diferentes actividades que lo componen. Por tanto, podríamos decir que

\footnotetext{
4 Calculado con datos de SADEI (Estadísticas Laborales y Comercio Exterior de Asturias) y el Instituto Nacional de Estadística (INE) (Estadística Estructural de Empresas).
} 
estamos ante un sector de sectores, compuesto por ramas productivas heterogéneas, por lo que es necesario diferenciarlo de otras dos agrupaciones con las que habitualmente es confundido:

a) el sector de bienes de equipo, que recoge otros servicios añadidos, como el diseño industrial, y que excluye la producción de bienes finales, así como la rama naval y la del automóvil;

b) el sector metal, que incluye todas las ramas metalmecánicas más la metalurgia básica. Actualmente, la integración hacia adelante de la siderurgia, con un mayor peso de los productos acabados y transformados, ha difuminado su frontera con "productos metálicos» ${ }^{5}$. En perspectiva histórica, se considera más apropiado tratar a la metalurgia como una entidad independiente, tanto por el peso autónomo de la siderurgia integral como por su particular andadura en el último siglo.

Se podría definir el sector metalmecánico como aquel conjunto de actividades transformadoras que, partiendo de la producción metalúrgica básica como input principal, se dedican a la fabricación de bienes destinados tanto a la demanda intermedia como, en menor medida, al consumo final.

En este trabajo se han homogeneizado los códigos CNAE: 74 y 93, que a su vez han tenido que ser armonizados con base en la clasificación específica utilizada en la contabilidad asturiana. La estructura sectorial que se utilizará en el resto del texto se recoge en la tabla 1 , utilizando tres niveles de división: sectores, subsectores y ramas de actividad.

\section{Tabla 1}

Composición de los sectores metalmecánico y metalúrgico por subsectores y ramas de actividad

\section{METALÚRGICO}

1. Productos siderúrgicos

2. Producción y primera transformación de metales no férreos

\section{METALMECÁNICO}

1. Productos metálicos

2. Maquinaria, equipo, electrónica y precisión

Maquinaria, equipo y material mecánico

Maquinaria, equipo y material eléctrico

Aparatos electrónicos, de radio, televisión y comunicaciones

Instrumentos de precisión, médicos, ópticos y relojería

3. Material de transporte

Fabricación de vehículos y sus componentes

Construcción y reparación naval

Otro material de transporte

Fuente: elaboración propia según CNAE-74, CNAE-93 y el sistema asturiano de clasificación regional.

\footnotetext{
${ }^{5}$ Una parte importante de los datos disponibles a partir de 1994 recogen como un único conjunto "Metalurgia y fabricación de productos metálicos», pudiendo distorsionar el análisis si esta puntualización no es tenida en cuenta.
}

El grueso de los datos utilizados para el presente trabajo proviene de SADEI (Sociedad Asturiana de Estudios Económicos y Empresariales). Esta sociedad pública, adscrita a la Consejería de Hacienda, es la fuente más rica y minuciosa para el estudio de la economía asturiana, pues se encarga de realizar, entre otras tareas, las tablas input-output, así como de proporcionar soporte metodológico en la elaboración de las cuentas regionales. La información obtenida del análisis de las mencionadas tablas se ha complementado con la recogida en otros informes y publicaciones, señalados en el apartado «Fuentes».

Al homogeneizar las tablas input-output se ha tenido en cuenta la introducción del modelo europeo contable SEC-95. Como consecuencia, las series relativas a producción y exportaciones, así como los diferentes indicadores, están recalculados al coste de los factores. Igualmente, se han ajustado utilizando el deflactor del VAB manufacturero propuesto por Prados de la Escosura con base 2010 (Prados de la Escosura, 2017). Cuando se haga referencia a una cifra en un año concreto, se hará en pesetas corrientes.

\section{La quiebra de los sectores industriales hegemónicos: un cambio de modelo productivo, 1970-1980}

Los años setenta marcarán un punto y aparte en la historia industrial. El final de la era Bretton-Woods y las crisis del petróleo pondrían de relieve la extenuación de un modelo de crecimiento que, si bien había marcado el desarrollo occidental durante las décadas pasadas, adolecía de profundas debilidades estructurales (Myro, 1989; Méndez, Sánchez y Benaul, 2003; Catalan, 2014). Ambos acontecimientos rubricaron el fin de la hegemonía del modelo industrial fordista, asociado a la gran empresa verticalmente integrada, que hacía descansar su fortaleza en el aprovechamiento de economías internas de escala con vistas a expandirse y alcanzar posiciones de liderazgo en un entorno que Chandler bautizó de «oligopolio global». La crisis del estándar imperante en la época dorada traería consigo la aparición de un nuevo paradigma: de un patrón de integración vertical se evolucionó hacia otro de especialización vertical, en el que cada país o región se orientaba, mediante la externalización, en fases específicas de la cadena de valor. Esta vía se favorecía de la reducción progresiva de los costes de transporte, así como de la liberalización y paulatina integración de los mercados mundiales, generándose los principales flujos comerciales entre países industrializados y con una tendencia intrasectorial (Feenstra, 1998; Hummels, Ishii y Yi, 2001).

Otra cuestión diferencial es que a partir de los años ochenta las crisis ya no inducirían a las pequeñas y medianas empresas a regresar a modelos de producción tradicionales, posponiendo sus esfuerzos de inversión, sino que estas intentarán encontrar una salida innovadora a través del desarrollo tecnológico y los proyectos llave en mano (Piore y Sabel, 1984). La cooperación entre agentes relacionados tomaría a partir de entonces especial relevancia, pues muchas de las empresas operantes en este nuevo paradigma no desarrollan su propia tecnología, por lo que su capacidad para integrar eficientemente los sistemas y componentes proporcionados por otros agentes se convertirá en un importante factor de competitividad (García Canal, 2017). Este cambio de pauta pondría en jaque a los grandes sectores asociados a la II Revolución Industrial, de los que la siderurgia representa quizá un ejemplo paradigmático (Piore y Sabel, 1984; Chandler, 1990, 1992). 
La siderurgia europea había entrado desde principios de la mencionada década en una situación de crisis generalizada. El sector se encontraba en plena fase expansiva, siguiendo las halagüeñas expectativas de consumo de acero a nivel global, basadas en las elevadas tasas de demanda que habían mostrado sus industrias de destino tradicionales - como la del automóvil- en los años anteriores. A partir de la primera mitad de los años setenta, estos mercados comenzaron a dar signos de saturación, lo que se traduciría en una reducción de la demanda de bienes de equipo, principal cliente siderúrgico (Piore y Sabel, 1984).

Por otro lado, la competencia en el mercado europeo se incrementaba al fortalecerse el sector en países que tradicionalmente habían tenido un peso relativamente menor en dicho escenario, como Italia y Holanda; al tiempo que progresivamente se hacía notar la presencia de países de reciente industrialización -NICs, newlly industrialized countries-, que operaban con estrategias de bajos precios, primero desde el Sudeste Asiático y, posteriormente, desde el Este de Europa (Bernabé, 1982; Pérez, 1994; Díaz Morlán, Escudero y Sáez, 2009). La escasa rentabilidad empresarial, sumada al proceso inflacionario general, redujo la tasa de inversión, crucial para el desarrollo de la industria primaria y transformadora, agravando de forma estructural la crisis del metal (Catalan, 2014).

Es en este contexto en el que han de enmarcarse los problemas que padecerá la industria asturiana que, desde sus orígenes, se había concentrado en torno a tres de los sectores que sufrirían más gravemente la reconversión industrial: la minería, la metalurgia y la construcción naval ${ }^{6}$. Al haber sido considerados como estratégicos por el régimen franquista, el período 1950-1970 había supuesto para ellos un importante auge. Con la decisión de localizar ENSIDESA en Avilés, Suanzes respondía a la misma llamada del carbón asturiano que, un siglo antes, habían sentido empresarios franceses, belgas y luxemburgueses (Nadal, 1975; Ojeda, 1985; Ocampo, 2004). Dentro de una opción que subordinaba la racionalidad económica a los intereses nacionales, la creación de ENSIDESA nacía de la necesidad de incrementar la producción de acero para abastecer al mercado doméstico. Para ello se crearía una gran siderurgia integral que desoligopolizara el sector sin necesidad de reducir los aranceles, y también como respuesta a la parsimonia inversora de los empresarios privados que, aletargados por la deriva proteccionista imperante desde finales del siglo pasado, se mostraban pesimistas en sus predicciones en cuanto a la demanda futura de acero en el país (Schwartz y González González, 1978; González González, 1988; Martín Aceña y Comín, 1991).

La crisis siderúrgica se manifestó en España con cierto retraso en comparación al resto de Europa, y la tardanza en la adopción de políticas estructurales de calado hizo que sus consecuencias fueran más largas y profundas. Debido a la propia coyuntura social de la época, la transición política se priorizó a la económica y, por el mismo motivo, se tardó mucho tiempo en tomar medidas de reestructuración que pudiesen quebrar la paz social (Navarro, 2004b; Arce, 2006). La política española de reestructuración terminaría por concentrar la si-

\footnotetext{
${ }^{6}$ El carbón, y con él la metalurgia, determinaron la especialización productiva de Asturias, siendo la base sobre la que se sustentaría el sector transformador, incluyendo la industria naval a partir del vapor. Para profundizar en el papel de estas actividades en la industrialización asturiana véase Nadal (1975), Ojeda (1985) y Ocampo y Suárez Cano (2018).
}

derurgia integral en el Principado, manteniendo únicamente una miniacería en Vizcaya -inaugurada en 1996 y previa fusión de Altos Hornos de Vizcaya (AHV) y ENSIDESA-, y clausurando la cabecera de Sagunto como consecuencia del plan de 1984 (Fernández de Pinedo, 2003; Díaz Morlán, Escudero y Sáez, 2008).

Centrándonos en el caso asturiano, con un sector industrial poco diversificado más allá de las tres ramas citadas, la entrada de la economía asturiana en la reconversión industrial no era muy esperanzadora. ENSIDESA mostraba a finales de los años setenta un claro problema de sobrecapacidad, así como una insostenible estructura de costes. La falta de racionalidad en la organización de la producción había reducido a mínimos los índices de productividad, muy alejados de los de aquellos países europeos que ya habían empezado años antes sus planes reconversores (Navarro, 2004a, 2004b; Vázquez, 2004). La decisión de potenciar el sector siderúrgico en la región y la posterior deriva hacia la unificación de la actividad integral en Asturias distaban mucho de la senda elegida por el País Vasco. Allí, el Gobierno autonómico apostaría abiertamente por una política diversificadora que impulsara nuevos sectores fabriles de alto componente tecnológico (Valdaliso, 2013).

\section{La respuesta del sector metalmecánico asturiano a la reconversión industrial}

\subsection{El impacto de los ajustes y la adhesión a la CEE, 1978-1990}

El Plan Siderúrgico Nacional de 1974, de marcado carácter expansivo, tuvo que ser cancelado por el Gobierno de la UCD en 1980, pues su sobreestimación del consumo doméstico de acero lo hacía inviable en el nuevo escenario (Pérez, 1994). Entretanto, el segundo shock petrolífero puso de manifiesto la necesidad de abordar un profundo proyecto reconversor, que afectaría a once actividades industriales en declive, entre las que destacaban la siderurgia y la construcción naval ${ }^{7}$. Pero ante la previsión del impacto negativo que tendría en el empleo y del malestar social que generaría en regiones como Asturias o País Vasco, la intervención prevista no fue llevada a cabo en profundidad. Tendría que ser el Gobierno socialista de Felipe González el que finalmente iniciase la reconversión, con Solchaga al frente del Ministerio de Industria y Energía ${ }^{8}$. Como sectores prioritarios, los ajustes sobre la siderurgia y la construcción naval se aplicarían a partir de 1984, siempre con la mirada puesta en la inminente entrada de España en la Comunidad Económica Europea (CEE) (Arce, 2006; Maluquer, 2014).

En materia de ocupación, el sector metalmecánico asturiano consiguió resistir razonablemente bien el envite de la primera intervención gubernamental, sustentado por el subsector «maquinaria, equipo, electrónica y precisión", que en 1990 presentaba cifras muy similares a las de 1978. Este hecho, sumado al decrecimiento generalizado del empleo industrial, se reflejó en un incremento del peso de la ocupación metalmecánica sobre el conjunto del sector secundario, así como en su mantenimiento con relación al total nacional (tabla 2). Este relativo

\footnotetext{
${ }^{7}$ Un tímido primer ajuste fue llevado a cabo según el RD 876/1981, en el que ya se identificaban las líneas maestras para la posterior reconversión. ${ }^{8}$ RDL 8/1983 de Reconversión y Reindustrialización, que daría pie a la posterior Ley $27 / 1984$.
} 
buen hacer no debe buscarse en la política pública de recolocación: además de promover la jubilación anticipada, el plan socialista crearía los Fondos de Promoción de Empleo (FPE), que no consiguieron favorecer el trasvase de empleo siderúrgico hacia la empresa privada operante en actividades transformadoras (Ruíz Valdepeñas, 1992). Buen ejemplo del fracaso de dicho plan es el impacto indirecto que la reconversión siderúrgica provocó durante esta década en la ocupación del subsector "productos metálicos", el más estrechamente relacionado con la industria matriz.

\section{Tabla 2}

Evolución de la ocupación en los subsectores metalmecánicos y el metalúrgico en Asturias (1978-2000)

\begin{tabular}{lccccc}
\hline & $\mathbf{1 9 7 8}$ & $\mathbf{1 9 8 5}$ & $\mathbf{1 9 9 0}$ & $\mathbf{1 9 9 5}$ & $\mathbf{2 0 0 0}$ \\
\hline Metalúrgico & 25.916 & 19.936 & 14.731 & 9.647 & 9.206 \\
\hline Metalmecánico & $\mathbf{2 2 . 4 7 5}$ & $\mathbf{1 8 . 7 1 0}$ & $\mathbf{1 8 . 0 2 9}$ & $\mathbf{1 4 . 1 9 0}$ & $\mathbf{1 8 . 0 6 2}$ \\
\hline $\begin{array}{l}\text { Productos } \\
\text { metálicos }\end{array}$ & 11.333 & 8.706 & 9.210 & 7.170 & 9.023 \\
\hline $\begin{array}{l}\text { Maquinaria, } \\
\text { equipo, } \\
\text { electrónica y } \\
\text { precisión }\end{array}$ & 5.799 & 5.707 & 5.581 & 4.756 & 6.488 \\
\hline $\begin{array}{l}\text { Material de } \\
\text { transporte }\end{array}$ & 5.343 & 4.297 & 3.238 & 2.264 & 2.551 \\
\hline $\begin{array}{l}\text { Metalmec. Ast./ } \\
\text { Metalmec. Esp. }\end{array}$ & $3 \%$ & $3 \%$ & $3 \%$ & $2 \%$ & $2 \%$ \\
\hline $\begin{array}{l}\text { Metalmec. Ast./ } \\
\text { Ind.Ast. }\end{array}$ & $17 \%$ & $19 \%$ & $21 \%$ & $20 \%$ & $29 \%$ \\
\hline
\end{tabular}

Fuente: elaboración propia a partir de SADEI, Tablas Input-Output, e INE, Anuarios Estadísticos de España.

Mención aparte merece la situación del subsector «material de transporte». Pese a que las ramas «fabricación de vehículos y sus componentes" $y$ "otro material de transporte» incrementaban sus plantillas -ocupando conjuntamente a 1370 activos en 1990-, su cifra total de ocupados caería arrastrada por la reconversión naval. Durante la aplicación del plan, entre 1984 y 1990 , los astilleros asturianos perdieron un $48 \%$ de sus efectivos totales, concentrándose el $70 \%$ de estas pérdidas en aquellos con más de 500 trabajadores, como el astillero público Juliana Constructora Gijonesa S. A. o el privado Duro Felguera ${ }^{10}$. A partir de entonces, algunos se verían abocados al cierre definitivo y otros buscarían su supervivencia a través de fusiones que en muchos casos terminarán por no ser viables en el largo plazo (Ocampo y Suárez Cano, 2018).

Tanto los ajustes navales como los siderúrgicos se vieron directamente determinados por la adhesión de España a la CEE

\footnotetext{
${ }^{9}$ El libro blanco de la reindustrialización (1983). MINER.

${ }_{10}$ Desde 1860, la actividad naval de Duro Felguera en Gijón derivaba del importante flujo comercial mantenido por la empresa con el País Vasco, donde se abastecía de hierro mientras aprovechaba los retornos del carbón y los hierros elaborados (Ocampo y Suárez Cano, 2018, p. 3).
}

y a la Comunidad Europea del Carbón y el Acero (CECA) en 1986, manifestando dos hechos: que las estimaciones y las intervenciones derivadas del plan de 1984 eran insuficientes y que la siderurgia española seguía sin ser competitiva en el mercado europeo (Navarro, 2004a). La actividad metalúrgica había seguido creciendo hasta 1985, momento en que se desplomaría a consecuencia de los ajustes derivados del plan aplicado a partir de 1984 y de su entrada en la Comunidad. Comercialmente, la adhesión de España a la CECA tendría un impacto muy significativo para el sector metalúrgico, ya que mientras las ventas españolas al mercado europeo estaban sujetas a una limitación voluntaria, las importaciones no podían ser restringidas. Así, mientras el consumo de acero foráneo por parte de la industria española aumentaba notablemente hasta 1990, las exportaciones siderúrgicas no dejaban de caer (Navarro, 2004b; Díaz Morlán y Sáez, 2019).

Pese a ser estos los años más duros de la reconversión, y a pesar del doble impacto - desde la oferta y la demanda - que supondría la caída de la metalurgia regional, el sector metalmecánico asturiano conseguiría engancharse al ciclo económico positivo de 1985-1990 y aprovechar el nuevo horizonte europeo para reorientar su producción (figura 1). Frente a la asfixiante situación de la siderurgia -cuyas exportaciones cayeron de los 170921 millones a los 88952 millones de pesetas en este período-, el sector transformador se mostraría muy dinámico en los mercados exteriores, hasta representar sus exportaciones el $18 \%$ del total industrial del principado en 1990.

"Productos metálicos» -subsector más importante en volumen dentro del metalmecánico y el más dependiente de la metalurgia - se vio muy afectado por el ajuste sufrido en ENSIDESA, lo que empeoraría levemente los resultados de todo el sector ${ }^{11}$. Entre 1978 y 1985, las ventas de "productos metálicos" a la metalurgia se verían reducidas en un $77 \%$, y ya nunca regresarían a su nivel inicial. Sin embargo, desde ese último año conseguiría reorientar su actividad hacia adelante, proveyendo de inputs a otras actividades, lo que explica su tendencia a converger con el resto de subsectores hasta el año noventa. En este caso, dicho viraje se orientaría hacia el mercado español -que representaba el $60 \%$ del total fabricado ese mismo añotras de un ligero repunte de sus exportaciones en 1985, cuando alcanzaron un $11 \%$ sobre su valor total producido, para estabilizarse en torno al $7 \%$ desde entonces.

Contrariamente, «maquinaria, equipo, electrónica y precisión» se vería favorecido por el incremento de la demanda por parte de la industria asturiana, incluyendo la importante inversión tecnológica en las plantas de ENSIDESA: a finales de los ochenta, las ventas en la región representaban un $63 \%$ de su fabricación total. Al mantenerse al alza la demanda regional, el subsector tenía escasos incentivos para competir más allá de nuestras fronteras. Además, a nivel desagregado se observa que la rama «maquinaria, equipo y material eléctrico» mostraba un comportamiento muy inestable, fluctuación que anulaba la tendencia positiva del conjunto, y contrastaba con la estabilidad de «maquinaria, equipo y material mecánico», que de forma constante destinaría en torno al $6 \%$ de su producción a mercados internacionales.

\footnotetext{
${ }^{11}$ La importancia de "productos metálicos» se explica por la tradición metalúrgica de la región, eslabón inmediatamente anterior a los transformados en la cadena de valor. Para comprobar cómo evoluciona el peso relativo de cada subsector sobre el conjunto, véase el anexo 1.
} 
El dato más sorprendente es el incremento de la actividad del subsector "material de transporte», que en 1990 se cifraba en 41130 millones de pesetas, un 14,2\% más que en 1978 . Como parte de este se estancaría la rama «construcción y reparación naval», frenada por una caída continuada de la demanda desde los años setenta, así como por una progresiva liberalización de los mercados, que estaba desplazando la contratación hacia astilleros internacionales con menores costes productivos (Valdaliso, 2003). Lo que se produjo en la construcción naval durante estos años fue un relevo empresarial, en virtud del cual los astilleros especializados en actividades de alto valor añadido reemplazarían a los gigantes públicos y privados de Gijón y Avilés. La permuta se concretó en el caso de los astilleros del occidente asturiano, Gondán y Armón, cuya participación en la actividad no dejaría de aumentar hasta ostentar entre ambos el 40,3\% de las entregas navales en Asturias entre 1987 y 1990 (Ocampo y Suárez Cano, 2018, p. 6).

Frente a los severos ajustes navales, la tendencia positiva del subsector "material de transporte» se debe al progreso de "fabricación de vehículos y sus componentes», así como al de "otro material de transporte», ya que en ambas ramas el valor de producción se duplicó hasta 1990. En la automovilística sería crucial la llegada de Suzuki a la región en 1984, cuando adquirió el 36,5\% del capital de la gijonesa Avello, empresa que terminaría por controlar completamente en 1988. La factoría de la compañía nipona, destinada a la fabricación de motocicletas de pequeña y gran cilindrada, contaba entonces con una capacidad máxima de fabricación anual de 63000 unidades (Avella, 1993). Igualmente relevante sería el papel de Armstrong Amortiguadores, que comenzó a operar en Asturias en 1976, cuando la firma británica adquirió Amortiguadores Bulnes. Tras ser proveedor principal de AUTHI en Pamplona, en los primeros años ochenta la planta asturiana se convertiría en suministrador de referencia para Ford en Valencia, fabricando los amortiguadores del modelo Fiesta. El crecimiento de la compañía la llevaría a ser absorbida en 1989 por la multinacional americana Tenneco, que la renombraría como Monroe Amortiguadores ${ }^{12}$.

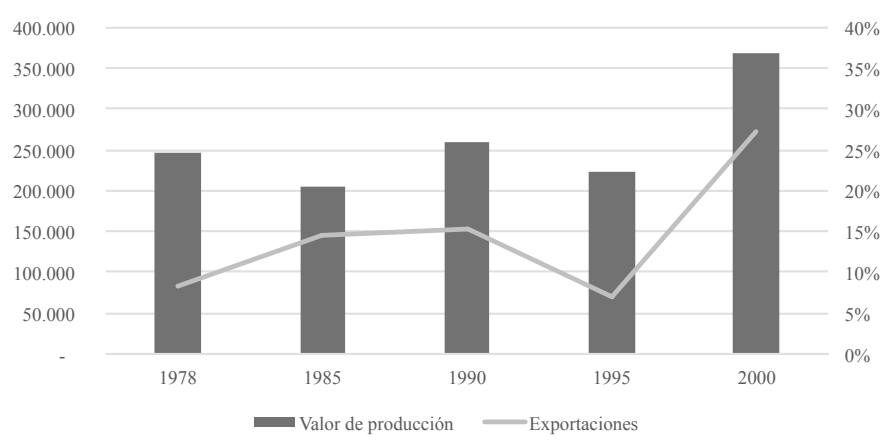

Figura 1. Evolución de las exportaciones metalmecánicas asturianas sobre el valor de producción, 1978-2000.

Fuente: elaboración propia con datos de SADEI, Tablas Input-Output. El valor de producción está expresado en millones de pesetas constantes de 2010. El peso de las exportaciones sobre el mismo está expresado en porcentaje.

\footnotetext{
${ }^{12}$ Para profundizar en la evolución de la actividad y la propiedad de la compañía, véase: www.vauste.es
}

El subsector "material de transporte» se mostraría como el más orientado hacia el mercado exterior: en 1990 le corresponderían 16446 millones de pesetas, el $40 \%$ del valor fabricado, frente al $19 \%$ de 1978 . Este crecimiento encaja con su dinámica productiva, anteriormente explicada. En primer lugar, "construcción y reparación naval» se erigió como la rama más destacada al final del período, con ventas al exterior por valor de 14572 millones de pesetas. Pese a la entrada en el mercado de competidores a bajo precio, provenientes principalmente del Sudeste Asiático, la orientación de los astilleros medianos hacia el nicho de embarcaciones bajo demanda permitiría que la construcción naval asturiana fuese cada vez más competitiva en el extranjero. Por último, la importancia del capital foráneo en la pequeña rama automotriz asturiana le otorgaba un carácter intrínsecamente internacional. Partiendo de unos niveles de exportación prácticamente nulos hasta 1985, «fabricación de vehículos y sus componentes» conseguiría triplicar sus ventas fuera de España en tan solo diez años.

El sostenimiento de la actividad metalmecánica durante los años más duros de la reconversión industrial la situaron en una posición de fuerza dentro de la estructura económica regional. Frente al inicio de la veloz terciarización de la economía asturiana y la crisis vivida por sus grandes sectores hegemónicos, el sector incrementaba su peso en el VAB industrial de la región, consiguiendo igualmente mantener su importancia con respecto al total del sector a nivel nacional (figura 2). Paralelamente, la productividad estimada por ocupado se incrementaba un $37 \%$ en el mismo período, siendo la tasa de variación para el conjunto de la industria asturiana de un $52 \%{ }^{13}$.

\subsection{El tejido metalmecánico en el más largo plazo: entre la crisis y el mercado global, 1990-2000}

Tras el ciclo económico positivo del quinquenio anterior, los primeros noventa traerían consigo una recesión generalizada que, en línea con la situación europea, provocaría una brusca contracción de la inversión industrial (Velasco y Plaza, 2003). Sin embargo, la creación del mercado único europeo en 1992, así como de la Organización Mundial del Comercio (OMC) en 1995 - acompañadas de nuevas devaluaciones- facilitaría la recuperación de las ventas al extranjero, favoreciendo que la segunda mitad de los noventa se tornara un período de importante expansión para la industria española (Maluquer, 2014; Catalan, 2019).

La recesión afectó duramente al sector metalúrgico europeo, que se vería comprometido por un incremento en el precio del petróleo fruto de la guerra del Golfo, así como por la entrada masiva de acero barato procedente de Europa Oriental a partir de 1991. La situación se agravaría en el caso español, por cuanto los ajustes siderúrgicos seguían a rebufo del resto de países, provocando que las medidas tomadas de cara a la incorporación al marco CECA se mostraran de nuevo insuficientes (Navarro, 2004b).

En tal escenario, ENSIDESA presentaba todavía unos altísimos costes productivos con relación a otras empresas. El repunte competitivo de finales de la década pasada parecía

\footnotetext{
13 Productividad aparente $=$ VABcf $/$ ocupados.
} 
un espejismo, pues en 1991 la acería registraba pérdidas históricas (Vázquez, 2004). El fracaso del intento reconversor condujo a la creación de la Corporación Siderúrgica Integral S. A. (CSI), resultante de la fusión de ENSIDESA y AHV previa intervención del Instituto Nacional de Industria (INI) y del Banco Industrial de Crédito. Aunque con matices, la creación de CSI ha sido generalmente considerada como un éxito, y en 1994 la corporación generaba de nuevo beneficios. Finalmente, y como parte de la política privatizadora del primer Gobierno de Aznar, en 1997 se constituirá Aceralia Corporación Siderúrgica S. A., con el grupo luxemburgués Arbed como socio mayoritario (Sierra, 2000; Navarro, 2004a; Catalan, 2014).

El plan CSI -que de nuevo recogía un duro ajuste de plantilla- tuvo repercusiones profundas sobre el empleo en el sector metalúrgico asturiano: tan solo entre 1990 y 1995 la rama siderúrgica perdería casi 5000 activos. A diferencia de lo ocurrido en el quinquenio 1980-1985, el metalmecánico no se salvaría esta vez de la recesión, si bien a finales de siglo conseguiría recuperar valores muy similares a los de 1990 , lastrado únicamente por las cifras del sector naval, castigado de forma directa por los ajustes reconversores. Esta recuperación colocaba al sector como referente industrial en materia de ocupación en una Asturias terciarizada, aunque sería menos acentuada respecto al metalmecánico nacional, que proporcionalmente había perdido muchos menos efectivos entre 1990 y 1995 (tabla 2). En este aspecto, los ajustes de «productos metálicos» se vieron recrudecidos por la convulsa situación siderúrgica, si bien conseguiría sobreponerse a partir de 1995. Por su parte, el descenso constante de "material de transporte» vendría determinado aún por los efectos de la reconversión naval.

De nuevo sería "maquinaria, equipo, electrónica y precisión» el subsector que mostrara la tendencia más estable, alcanzando niveles históricos de ocupación en el año 2000. Esta dinámica estuvo liderada por «maquinaria, equipo y material mecánico», que apenas sufrió los efectos de la crisis y en este último año contaba con 3649 ocupados. Por su parte, la rama "maquinaria, equipo y material eléctrico» sí se vería afectada por la recesión, aunque se recuperaría posteriormente hasta cifras casi idénticas a las de 1990.

La producción global del sector metalmecánico mostró una clara dinámica de rebote, ya que tras el desplome provocado por la recesión, su actividad repuntaría notablemente en el último quinquenio del siglo xx. Este incremento estuvo especialmente espoleado por el aumento de la demanda intermedia a nivel global, hecho del que conseguiría favorecerse notablemente el sector asturiano (figura 1). El Ministerio de Industria ya identificaba en 1995 la apertura exterior como una posible arma de doble filo, pues mientras los bienes de equipo españoles se posicionaban como un segmento de gran especialización, el pequeño tamaño medio de las empresas dificultaba su acceso a economías internas de escala ${ }^{14}$.

Si bien en el plano internacional, y al menos en el corto plazo, el sector metalúrgico no logró cumplir el objetivo de expansión marcado con la creación de Aceralia -entre 1995 y 2000 las ventas del conjunto al extranjero se redujeron un $12 \%$-, el crecimiento metalmecánico al final de los noventa

\footnotetext{
${ }^{14}$ El Libro blanco de la industria: una política industrial para España (1995).
} MINER. radicó en gran medida en la capacidad que tuvieron sus diferentes subsectores para aprovechar la integración de los mercados mundiales. En el año 2000, sus exportaciones representaban ya el $28 \%$ del total de la industria regional, un $10 \%$ más que en 1990.

Las cifras productivas del subsector "productos metálicos" decrecerían ligeramente durante la crisis, para recuperarse a partir de 1995 orientadas hacia el mercado nacional. El posicionamiento internacional del subsector resultaba comparativamente más complicado al operar este en un nicho de mercado en que las estrategias de bajo precio eran más viables que la especialización. El incremento de sus ventas al exterior, aunque notable, resultó menos pronunciado que en aquellas ramas altamente especializadas. Entre los años 1990 y 2000 sus ventas quedaron confinadas al mercado interior, representando sus exportaciones un máximo del $11 \%$ sobre la producción total en el año 2000, valores equiparables a los logrados en 1985.

Por su parte, la actividad de «maquinaria, equipo, electrónica y precisión" conseguiría mantenerse estable y al alza durante la recesión, tendencia sostenida desde 1978. El subsector despegará finalmente a partir de 1995, impulsado a partes iguales por las ramas mecánica y eléctrica, que crecerán en torno al $70 \%$ hasta el año 2000 . El gran avance del subsector se debió a la focalización de sus capacidades en rebasar sus mercados tradicionales. Al desagregar los destinos de su actividad, puede observarse cómo el peso de la demanda asturiana y española sobre sus ventas se estabilizó, respectivamente, en torno al $40 \%$ en el último quinquenio, mientras las exportaciones alcanzaban los 16945 millones de pesetas al final del mismo período, cerca de un $20 \%$ de su valor de producción total. En este sentido, la instalación del grupo alemán Thyssenkrupp en la región, en 1992, parecía un buen reflejo de la confianza en el potencial industrial asturiano. Bajo la denominación Thyssenkrupp Norte S. A., la compañía emplazó en Mieres una planta destinada a la fabricación de cintas y escaleras mecánicas, localización a la que se sumaría en 1996 la planta Airport Solutions, especializada en pasarelas aeroportuarias ${ }^{15}$.

El subsector "material de transporte» sería el más castigado en materia productiva por la recesión de principios de los noventa. En la rama de la construcción naval, el estancamiento de los pedidos durante la década anterior, así como los ajustes derivados de la reconversión, hundirían la actividad hasta 1995. Pese a todo, la nueva base empresarial liderada por los astilleros del occidente, junto a una rama automovilística pequeña pero dinámica, conseguirán hacer resurgir al subsector: en el año 2000 el valor final de la producción agregada de «material de transporte» se incrementaba un $18,6 \%$ respecto a 1990. Esta evolución responde a la línea de especialización ya perfilada en el período anterior. Si la rama "construcción y reparación naval» siempre había tenido una mayor orientación internacional, este rasgo se acentuó con el liderazgo ejercido por los astilleros medianos, que al final del período destinaban a los mercados exteriores el $78 \%$ de su actividad. La rama del automóvil siguió la misma tendencia con un $56,7 \%$, aunque en este caso el mercado nacional pesaba mucho más que el regional en el porcentaje restante.

\footnotetext{
${ }^{15}$ Paralelamente, la empresa instaló un centro de I+D+i en Gijón en 1996 (La Nueva España, varias fechas).
} 


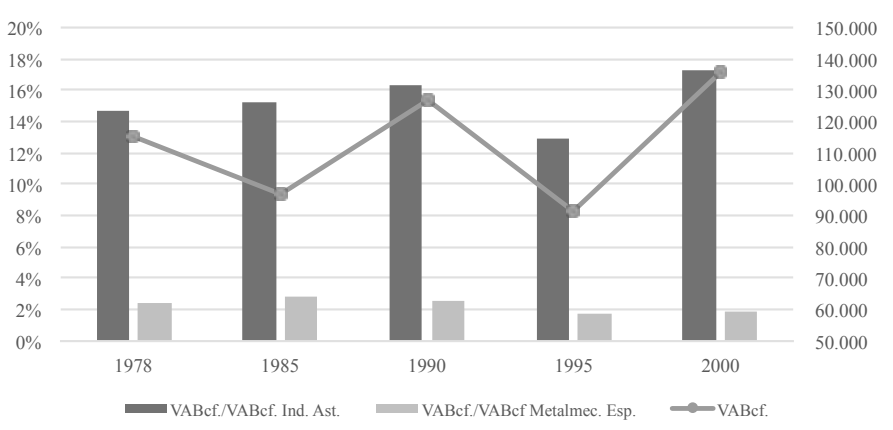

Figura 2. Evolución del VABcf metalmecánico en Asturias respecto al total de la industria asturiana y al sector en España, 1978-2000.

Fuente: elaboración propia a partir de SADEI, Tablas Input-Output, e INE, Anuarios Estadísticos de España. El valor absoluto del VABcf está expresado en millones de pesetas constantes de 2010 . Su peso sobre las otras dos variables está expresado en porcentaje.

En cómputo global, la todavía difícil situación de la siderurgia agravaría el efecto que la recesión de la economía española ejerció sobre el sector metalmecánico en Asturias. Este hecho lo sumiría en una profunda crisis durante el primer quinquenio de los años noventa, de la que, sin embargo, conseguiría sobreponerse rápidamente en la segunda mitad de la década. Esta brusca caída dañaría ligeramente su posición con relación al conjunto del sector a nivel nacional -oscilando, pese a ello, en cifras muy similares a las mostradas anteriormente, entre un $2 \%$ y un $3 \%$ - ya que, a nivel agregado, este último aún conseguiría crecer ligeramente durante estos años. Pese a ello, a nivel regional el metalmecánico se consolidaría como referente manufacturero, incrementando su importancia en una economía industrial en declive y en rápida transición hacia la terciarización (figura 2). Como apunte final cabe destacar el papel que el buen hacer del subsector «maquinaria, equipo, electrónica y precisión" pareció repercutir sobre el total de la industria, especialmente desde el mecanizado, incrementándose la productividad aparente sobre la ocupación del sector fabril en un 39\% en la última década.

\section{El cluster metalmecánico de Asturias}

Al inicio de la reconversión industrial la distribución geográfica de la actividad metalmecánica en Asturias era un claro reflejo del devenir histórico de la industria en la región: desde los focos originarios de los valles hulleros del Nalón (Langreo) y el Caudal (Mieres), esta había pasado a concentrarse en Gijón y Avilés tras la implantación de ENSIDESA. A estos concejos debemos sumar Oviedo, que supuso igualmente un importante polo de atracción tanto por su privilegiada posición geográfica como por el efecto capitalidad. Dichos municipios estructuran el conocido como ocho asturiano, eje vertebrador de la región, organizado como un sistema metropolitano polinuclear -de cuya influencia se favorecieron otros concejos cercanos-, y que ofrecía a las empresas la posibilidad de beneficiarse de las externalidades y rentas de situación generadas por el desarrollo industrial posterior a 1950 (Benito del Pozo, 1993). Esta pauta se refleja en la evolución de concejos colindantes con Gijón, Avilés y Oviedo frente al decaimiento de las cuencas mineras (figura 3 ).

A lo largo del período el sector se estructuró en torno a una base conformada por empresas medianas, entre los 100 y 200 empleados, cuya relevancia estratégica las haría consolidarse como tractoras por su efecto dinamizador sobre el resto ${ }^{16}$. Esta evolución responde a un proceso de reorganización jerárquica: ante el declive de las grandes empresas en torno a las cuales se había concentrado la actividad en las décadas anteriores, emergían ahora nuevos actores que tomaban su testigo, impulsados por las capacidades acumuladas durante décadas a la sombra de aquellas (Catalan, Miranda y Ramon-Muñoz, $2011)^{17}$.

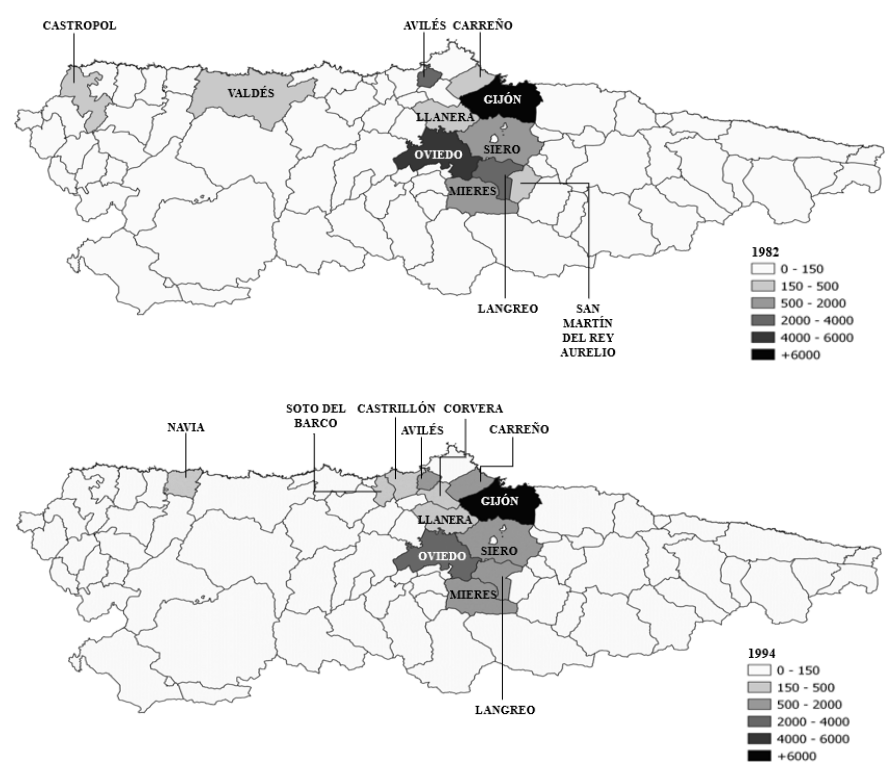

Figura 3. Distribución por concejos del número de activos en el sector metalmecánico asturiano, 1982 y 1994.

Fuente: elaboración propia con datos de SADEI, La Renta de los Municipios Asturianos. Años extremos elegidos según disponibilidad de datos.

Como apunte complementario a esta aglomeración, cabe destacar que los coeficientes de especialización regional en materia de ocupación superaban la media española, tanto al inicio como al fin de la reconversión industrial, si bien es cierto que debido a la rápida terciarización asturiana el nivel decrece ligeramente: de 1,31 en 1978 a 1,11 en el $2000^{18}$.

Aun así, la concentración geográfica es un factor necesario pero no suficiente para la identificación de un cluster. Según la definición de Porter mencionada en el apartado 1, las empresas y organizaciones que lo conformen deben estar relacionadas entre sí, bien sea a través de flujos comerciales bien a través de conexiones intangibles en materia de innovación y desarrollo. Cabe apuntar que esta conjunción tiende a ser positiva, de forma que las industrias con altos valores de relación comercial muestran habitualmente un patrón similar en innovación y cooperación (Navarro, 2003).

\footnotetext{
${ }^{16}$ En volumen de empleo predominarían las empresas de entre 1 y 50 empleados, que en el año 2000 concentraban el $44 \%$ de los activos.

17 Véase anexo 2.

18 Índice de especialización regional con relación al empleo del sector en el conjunto de España, expresado mediante la fórmula $\mathrm{Ce}=(\mathrm{Eir} / \mathrm{Er}) /(\mathrm{Ei} / \mathrm{E})$, donde Eir hace referencia al empleo del sector $i$ en la región $r$, Er es el empleo total de la región, Ei es el empleo sectorial en el país y E es el empleo total del país. Un valor superior a 1 indica mayor peso relativo del sector en el empleo de la región que en el conjunto del país.
} 
Los coeficientes técnicos entre los subsectores metalmecánicos y la metalurgia muestran - sin tener en cuenta las ramas energéticas, cuya producción es transversal como input a cualquier actividad- los valores más altos de la economía industrial asturiana ${ }^{19}$. Asimismo, nos ofrecen una imagen de la evolución de la cadena de valor metalmecánica en la región (tabla 3).

\section{Tabla 3}

Coeficientes técnicos regionales de los subsectores metalmecánicos y el sector metalúrgico, 1978 y 2000

\begin{tabular}{lcccc}
\hline $\begin{array}{l}1978 \\
\text { Compras } \\
\text { Ventas }\end{array}$ & Metalúrgico & $\begin{array}{c}\text { Mroductos } \\
\text { metálicos }\end{array}$ & $\begin{array}{c}\text { Mainaria, } \\
\text { equipo, } \\
\text { electrónica y } \\
\text { precisión }\end{array}$ & $\begin{array}{c}\text { Material } \\
\text { de } \\
\text { transporte }\end{array}$ \\
\hline Metalúrgico & 0,011 & 0,197 & 0,054 & 0,028 \\
\hline $\begin{array}{l}\text { Productos } \\
\text { metálicos }\end{array}$ & 0,029 & 0,043 & 0,075 & 0,048 \\
\hline
\end{tabular}

Maquinaria,

equipo,

electrónica

0,011

0,009

0,070

0,015

y precisión

Material de

transporte

\begin{tabular}{|c|c|c|c|c|}
\hline $\begin{array}{l}2000 \\
\text { Compras } \\
\text { Ventas }\end{array}$ & Metalúrgico & $\begin{array}{l}\text { Productos } \\
\text { metálicos }\end{array}$ & $\begin{array}{l}\text { Maquinaria, } \\
\text { equipo, } \\
\text { electrónica y } \\
\text { precisión }\end{array}$ & $\begin{array}{c}\text { Material } \\
\text { de } \\
\text { transporte }\end{array}$ \\
\hline Metalúrgico & 0,036 & 0,185 & 0,065 & 0,037 \\
\hline $\begin{array}{l}\text { Productos } \\
\text { metálicos }\end{array}$ & 0,004 & 0,025 & 0,039 & 0,027 \\
\hline $\begin{array}{l}\text { Maquinaria, } \\
\text { equipo, } \\
\text { electrónica } \\
\text { y precisión }\end{array}$ & 0,022 & 0,017 & 0,048 & 0,031 \\
\hline
\end{tabular}

Material de

transporte

0,048

Fuente: elaboración propia con datos de SADEI, Tablas Input-Output. Leído en vertical, las cifras expresan la demanda que la rama $j$ realiza de la rama $i$ para la producción de una unidad. En horizontal, las ventas de la rama $i$ a la rama $j$ para la producción de una unidad.

La lectura más informativa del cuadro se realiza desde el punto de vista de la demanda ("compras», fila superior de la tabla). Se observa que el desarrollo del sector metalmecánico incrementó ligeramente la demanda que sus subsectores hacían de la producción metalúrgica regional. La excepción sería el caso de "productos metálicos», que se vería afectado por la integración hacia delante de la cadena de valor siderúrgica, hasta solaparse en ciertos eslabones con su propia actividad,

${ }^{19}$ El coeficiente técnico regional viene dado por la expresión $\mathrm{Arij}=\mathrm{Xrij} / \mathrm{Xj}$, donde Xrij expresa el consumo de bienes o servicios producidos en la región por la rama $i$ y utilizados en su producción por la rama $j$, y Xj es el valor total de la producción de la rama $j$. lo que a su vez explica el incremento de inputs reutilizados por la metalurgia.

La importancia de "productos metálicos» como proveedor intermedio - cada vez más destinado al mercado nacional, como se apuntaba anteriormente- se redujo considerablemente en favor de la maquinaria ${ }^{20}$. La demanda que todos los subsectores harían de «maquinaria, equipo, electrónica y precisión» se incrementó durante este período, aumentando asî la importancia que su actividad ejercería sobre el resto. En este caso, la excepción se daría en la reutilización de su propia producción, disminuida por la progresiva implementación de tecnología y maquinaria foránea en su actividad. Por último, y de forma lógica, la contribución de "material de transporte" al desarrollo del resto de actividades es nula, si bien destaca el repunte de la producción para su propia reutilización sectorial, debido a su paulatina especialización en fases intermedias de la cadena productiva. En resumen, la dinámica global del sector encajaría con el patrón de un metalmecánico dinámico y que evolucionaba técnicamente, por lo que dependía cada vez de más y mejor maquinaria para continuar desarrollándose.

Este avance técnico no vendría impulsado únicamente desde el interior de la industria. A partir de los años ochenta, la vertiente reindustrializadora de la reconversión propondría una política de promoción de suelo industrial que promoverá gran parte de los polígonos industriales y parques tecnológicos actualmente existentes en la región, en los que el metalmecánico fue siempre el sector predominante (Benito del Pozo, 2014). Especialmente relevantes serían los espacios dedicados a la implantación de agentes tecnológicos y del conocimiento, cuya relación con las empresas del sector metalmécanico ha sido siempre muy estrecha, actuando como agentes facilitadores en el avance hacia un modelo de fabricación avanzada.

En este sentido, la creación del Parque Tecnológico de Asturias en 1991 (Llanera) supuso un punto de inflexión en la apuesta de la región por el desarrollo tecnológico ligado al sector industrial. Desde su fundación, el espacio -en que conviven empresas y organizaciones transversales, tanto de carácter público como privado- ha estado claramente dominado por actividades relacionadas con las TIC, la ingeniería y los bienes de equipo de alto componente tecnológico. Cabe destacar la implantación ese mismo año de la sede de la Fundación ITMA (Instituto Tecnológico de Materiales de Asturias), orientada a la investigación e innovación basada en materiales, donde el acero tendrá un papel destacado ${ }^{21}$.

No menos importante sería la creación en el año 2000 del Parque Científico-Tecnológico de Gijón, centro neurálgico de la Milla del Conocimiento Margarita Salas ${ }^{22}$. El complejo fue proyectado en torno al campus gijonés de la Universidad de Oviedo, sede de los principales estudios técnicos ofertados en la región, y que desde 1994 acogería al Instituto Universitario

\footnotetext{
${ }^{20}$ La caída de la demanda de productos metálicos por parte de la industria tiene su contraparte en su incremento por parte de la construcción. El coeficiente técnico regional entre "productos metálicos" y "construcción" pasa de 0,020 en 1978 a 0,126 en el año 2000.

${ }^{21}$ En 2005, la Fundación crearía el Centro Tecnológico del Acero y Materiales Metálicos en Avilés. Véase: www.itma.es

22 Desde 2004 el espacio acogería a su vez a la Fundación PRODINTEC, agente tecnológico especializado en la potenciación de la industria asturiana a través de la innovación en procesos de fabricación y productos.
} 
de Tecnología Industrial de Asturias (IUTA), del que junto a la Universidad y otros agentes de conocimiento asociados forman parte como colaboradoras las empresas más importantes del sector metalmecánico de la región ${ }^{23}$.

Los facilitadores tecnológicos y centros de conocimiento, junto con distintos entes públicos como el Instituto de Desarrollo Económico del Principado de Asturias (IDEPA, creado en 2002 y heredero del antiguo Instituto de Fomento Regional) o Asturex (Sociedad de Promoción Exterior del Principado de Asturias, fundada en 2005) serían una pieza clave en la red que, en 2016 e impulsada por la patronal del metal en Asturias (FEMETAL), terminaría constituyéndose como MetaIndustry4, Cluster de Fabricación Avanzada, que en 2019 contaba con 57 asociados. Seis años antes, aunque en este caso impulsado por el gigante siderúrgico Arcelor Mittal, surgiría el Polo del Acero, conformado por 18 organizaciones ${ }^{24}$.

La apuesta del metalmecánico por la innovación se reflejaba igualmente en el mayor esfuerzo relativo que este sector realizaba en materia de inversión en $I+D+i$ en comparación con el resto de la economía asturiana: en el año 2000 el conjunto representaba el $44,8 \%$ del gasto total regional en esta materia ${ }^{25}$. Como apunte final, es destacable que en 1998 Asturias fuese la cuarta región española con mayor número de empresas ligadas a actividades de ingeniería y con una facturación superior a los 3000 millones de pesetas, solo por detrás de Madrid (25), Vizcaya (11) y Barcelona (6) (Méndez, Sánchez y Benaul, 2003, p. 487).

\section{Conclusiones}

En este trabajo se ha revisado la visión tradicional mantenida sobre la reconversión industrial en España, que la emplazaba como punto final para el tejido fabril asturiano. La metalurgia básica, heredera de los yacimientos hulleros, había sido un pilar estratégico de la economía asturiana desde mediados del siglo xix y, muy especialmente, desde la creación de ENSIDESA. La concentración de actividades transformadoras en torno a la siderurgia propició la aparición de ventajas competitivas alejadas del carbón, que el sector metalmecánico supo aprovechar tras el declive de las industrias hegemónicas. Esta aglomeración derivaría en la generación de un entorno clusterizado, que permitió a un grupo de medianas empresas - tanto asturianas como extranjeras y con un marcado perfil exportador- convertirse en referentes industriales para Asturias.

Ante la caída de ENSIDESA, "productos metálicos» conseguirá reorientarse hacia el aprovisionamiento de bienes intermedios para diversas actividades, contando con el mercado español como principal destino. Por el contrario, "maquinaria, equipo, electrónica y precisión" concentraría sus esfuerzos en abastecer la creciente demanda de maquinaria por parte de otras ramas asturianas que vinculaban sus posibilidades de supervivencia a la necesidad de moderni-

\footnotetext{
${ }^{23}$ Para el listado completo de colaboradores, véase: www.iuta.uniovi.es

${ }^{24}$ En 2001 Aceralia, Arbed y Unisor crearían el Grupo Arcelor, que en 2006 se fusionaría a su vez con Mittal Steel. ArcelorMittal Asturias sigue siendo la única planta siderúrgica integral de España. En 2018 contaba con una plantilla que rondaba los 5400 empleados y su presencia sigue siendo clave para la región, pues de ella dependen todavía muchas empresas metalmecánicas consideradas como auxiliares.

25 INE, Encuesta sobre innovación tecnológica en las empresas (2000).
}

zarse. Sobre esta base, desde 1995 el subsector conseguirá dar el salto a los mercados internacionales a partir de una oferta que, a través de la innovación, progresivamente se especializaría en pedidos bajo demanda y de alto componente tecnológico.

Un sendero muy similar recorrería el subsector «material de transporte», aquel con mayor presencia relativa en el extranjero. En este sentido destaca la construcción naval, cuya competitividad exterior descansará sobre una nueva base empresarial focalizada en actividades de alto valor añadido (buques de apoyo, embarcaciones de recreo y suministro a la industria off-shore).

De cara al futuro, será conveniente profundizar en la caracterización de dicho cluster, así como en su repercusión sobre el global de la economía asturiana. Igualmente, será de interés ampliar el escenario estudiado, llevándolo al análisis comparativo con otras regiones europeas que partiesen de unas ventajas comparativas similares, de forma que se puedan valorar las vías de salida seguidas por las regiones siderúrgicas en declive a partir de los años setenta.

\section{Agradecimientos}

Este trabajo cuenta con la financiación del Ministerio de Universidades a través de una beca FPU adscrita al proyecto «¿Capitalismo mediterráneo? Éxitos y fracasos del desarrollo industrial en España, 1720-2020» (PGC2018-093896-B-I00).

Quiero expresar mi agradecimiento a los profesores Jordi Catalan y Marc Prat, directores de la tesis en que se enmarca este trabajo, por su dedicación y sus valiosas enseñanzas, así como al profesor Joaquín Ocampo por su apoyo y por compartir generosamente conmigo su sabiduría. Igualmente quisiera dar las gracias a los revisores de Investigaciones de Historia Económica, que han contribuido enormemente a mejorar la primera versión de este trabajo, y a los profesores Miquel Gutiérrez, Anna Aubanell, Yolanda Blasco y Vittorio Galletto por sus comentarios sobre el mismo. Gracias también a Guillermo Ulacia por su confianza constante, a Ramiro Lomba y Javier Galán de SADEI, que me facilitaron el acceso al grueso de los datos que componen este estudio, y a José Luis Rebordinos por abrirme las puertas de la hemeroteca de LNE.

\section{Fuentes}

BOE (Boletín Oficial del Estado). Disponible en: www.boe.es Hemeroteca del diario La Nueva España, Oviedo.

INE

Anuarios estadísticos de España 1978-2000. Disponible en: www.ine.es.

Encuesta sobre innovación tecnológica en las empresas, 2000.

MINER

El libro blanco de la reindustrialización, 1983.

El libro blanco de la industria: una política industrial para España, 1995.

Fondo documental de SADEI Cuentas regionales de Asturias y tablas Input-Output (19782000).

Renta de los municipios asturianos (1980-2000). La industria siderometalúrgica en Asturias (Informe realizado junto a NEI, 1971). 


\section{Bibliografía}

Arce, J. M. (2006) «La fase dura de la reconversión industrial: 19831986», Historia del Presente, 8, 61-101.

Avella, L. (1993) «Producción ajustada: la experiencia de Suzuki Motor España en Asturias», Revista Europea de Dirección y Economía de la Empresa, 2 (3), 129-140.

Benito del Pozo, P. (1993) "Metrópoli e industria en Asturias», en García Delgado, J. L. et al. (eds.). Economía y empresa en Asturias : homenaje a Ignacio Herrero Garralda, marqués de Aledo. Madrid: Editorial Civitas.

Benito del Pozo, P. (2014) «Políticas públicas y transformación del paisaje industrial en Asturias: de la gran fábrica a las áreas empresariales», en Benito del Pozo, P. (ed.). Planificación territorial y desarrollo del suelo empresarial en España. Pamplona: Thomson Reuters.

Bernabé, J. (1982) «Los problemas de reestructuración de la siderurgia en la década de los ochenta y sus repercusiones en el proyecto de la IV Planta Siderúrgica Integral de Sagunto», Cuadernos de geografía, 31, 145-160.

Catalan, J. (1995) «Sector exterior y crecimiento industrial: España y Europa (1939-59)», Revista de Historia Industrial, 8, 99-146.

Catalan, J. (2014) «From the Great Depression to the Euro Crisis, 19292013: A Global Approach", Revista de Historia Industrial, 56, 15-45.

Catalan, J. (2017) «The Life-Cycle of the Barcelona Automobile-Industry Cluster, 1889-2015», Revista de Historia Industrial, 26 (66), 77-125.

Catalan, J. (2019) «La industrialización de Cataluña, 1685-2018: el papel de las políticas industriales», en García Ruíz, J. L. (ed.). Políticas industriales en España. Pasado, presente y futuro. Madrid: Paraninfo, pp. 157-198.

Catalan, J., Miranda, J. A. y Ramon-Muñoz, R. (2011) «Distritos, clusters y ventaja competitiva: interpretaciones y debates», en Catalan, J., Miranda, J. A., y Ramon-Muñoz, R. (eds.). Distritos y clusters en la Europa del Sur. Madrid: LID Editorial Empresarial, pp. 9-34.

Chandler, A. D. (1990) Scale and scope: The Dynamics of Industrial Capitalism. Cambridge: Harvard University Press.

Chandler, A. D. (1992) «Organizational Capabilities and the Economic History of the Industrial Enterprise», The Journal of Economic Perspectives, 6 (3), 79-100.

Díaz Morlán, P., Escudero, A. y Sáez, M. A. (2008) «El desmantelamiento de la siderurgia integral del Mediterráneo español (19771984)», Revista de Historia Industrial, 38, 161-188.

Díaz Morlán, P., Escudero, A. y Sáez, M. A. (2009) «The restructuring of the Spanish integrated steel industry in the European panorama (1971-1986): A lost opportunity», Business History, 51(4), 547-568.

Díaz Morlán, P. y Sáez, M. A. (2019) «Estado, industrialización y desindustrialización. Las políticas siderúrgicas españolas en la segunda mitad del siglo xx», Revista de Historia Industrial, 29 (75), 133-168.

Díez-Minguela, A., Martínez-Galarraga, J. y Tirado, D. (2018) Regional Inequality in Spain, 1860-2015. London: Palgrave Macmillan.

Ducoing, C. y Tafunell, X. (2013) «Formación bruta de capital en bienes de equipo en Chile, 1856-1930. Fuentes nacionales y extranjeras", America Latina en la Historia Economica, 20 (1), 5-34.

Eaton, J. y Kortum, S. (2001) «Trade in capital goods», European Economic Review, 45 (7), 1195-1235.

Feenstra, R. C. (1998) «Integration of Trade and Disintegration of Production in the Global Economy", Journal of Economic Perspectives, 12 (4), 31-50.

Fernández de Pinedo, E. (2003) «Desarrollo, crisis y reconversión de la siderurgia española a través de una empresa vizcaína, AHV (1929-1996)», Ekonomiaz: Revista Vasca de Economía, 54, 28-51.

García Canal, E. (2017) «Riesgo tecnológico y relacional y desarrollo de las empresas contratistas llave en mano: el caso de Astilleros Gondán en la construcción naval», Universia Business Review, 53, 60-107.

González González, M. J. (1988) «Minería, siderurgia y empresa pública en Asturias: el siglo XX", en Hernández Marco, J. L. y Fernández de Pinedo, E. (eds.). La industrialización del norte de España. Barcelona: Universidad del País Vasco y Crítica, pp. 128-146.
Hummels, D., Ishii, J. y Yi, K. M. (2001) "The nature and growth of vertical specialization in world trade», Journal of International Economics, 54 (1), 75-96.

Landes, D. (1969) The Unbound prometheus : technological change and industrial development in Western Europe from 1750 to the present. Londres: Cambridge University Press.

Maluquer, J. (2014) La economía española en perspectiva histórica. Barcelona: Pasado \& Presente.

Marshall, A. (1963) Principios de Economía. Un tratado de introducción. Madrid: Aguilar (ed. original en inglés, 1890: Principles of Economics. Londres: Macmillan and Co.).

Martín Aceña, P. y Comín, F. (1991) INI, 50 años de industrialización en España. Madrid: Espasa Calpe.

Martínez-Galarraga, J., Rosés, J. y Tirado, D. (2015) «The long-term patterns of regional income inequality in Spain, 1860-2000", Regional Studies, 49 (4), 502-517.

Méndez, R., Sánchez, S. y Benaul, J. M. (2003) «Crisis, reconversión e integración en Europa, 1975-2000», en Benaul, J. M., Sudrià, C. y Nadal, J. (eds.). Atlas de la industrialización de España: 1750-2000. Barcelona: Crítica, pp. 389-554.

Myro, R. (1989) «La industria: expansión, crisis y reconversión», en García Delgado, J. L. (ed.). España, economía. Madrid: Espasa Calpe, pp. 197-231.

Nadal, J. (1975) El fracaso de la Revolución Industrial en España. Barcelona: Ariel.

Navarro, M. (2003) «Análisis y políticas de clusters teoría y realidad», Ekonomiaz: Revista Vasca de Economía, 53, 14-49.

Navarro, M. (2004a) «La larga marcha de la siderurgia española hacia la competividad», Economía Industrial, 355-356, 167-184.

Navarro, M. (2004b) «La reconversión de la siderurgia española entre 1975 y 1995. Una azarosa andadura con un final feliz», en González González, M. J. (ed.). Hierro y acero ante la mundialización: una perspectiva histórica. Avilés: Aceralia, pp. 145-160.

Ocampo, J. (2004) «Hierro y acero en los orígenes de la industrialización asturiana», en González González, M. J. (ed.). Hierro y acero ante la mundialización: una perspectiva histórica. Avilés: Aceralia, pp. 35-53.

Ocampo, J. y Suárez Cano, P. (2018) «Pequeños, familiares y competitivos: astilleros y construcción naval en Asturias (c. 1750-2015)», Investigaciones de Historia Economica, 14 (1), 43-53.

Ojeda, G. (1985) Asturias en la industrialización española, 1833-1907. Madrid: Universidad de Oviedo, Servicio de Publicaciones.

Pérez, M. A. (1994) «La segunda reconversión siderúrgica», en Ojeda, G. y Vázquez, J. A. (eds.). Historia de la Economía Asturiana. Oviedo: La Nueva España, pp. 833-848.

Piore, M. J. y Sabel, C. F. (1984) The second industrial divide: possibilities for prosperity. Nueva York: Basic Books.

Pollard, S. (1981) Peaceful conquest: the industrialization of Europe, 1760-1970. Oxford: Oxford University Press.

Porter, M. (1998) "Clusters and the New Economics of Competition», Harvard Business Review, 76 (6), 77-90.

Porter, M. y Ketels, C. (2009) «Cluster and Industrial Districts: Common Roots, Different Perspectives», en Becattini, G., Bellandi, M. y De Propis, L. (eds.). Handbook of Industrial Districts. Northampton: Edward Elgar Publishing, pp. 172-187.

Prados de la Escosura, L. (2017) Spanish economic growth, 1850-2015. Londres: Palgrave Macmillan.

Ruíz Valdepeñas, H. P. (1992) «Reconversión y reestructuración industrial en Asturias», ERÍA: Revista cuatrimestral de geografía, 28, 151-164.

Sanchís, M. T. y Cubel, A. (2007) «Comercio de bienes de capital y desarrollo de la industria de bienes de equipo en España, 19501975», en Pascual, P. y Fernández, P. (eds.). Del metal al motor. La industria de transformados metálicos en España. Bilbao: Fundación BBVA, pp. 157-192.

Schwartz, P. y Gónzalez González, M. J. (1978) Una historia del Instituto Nacional de Industria (1941-1976). Madrid: Tecnos.

Sierra, M. P. (2000) «Reconverisón e internacionalización de la siderurgia integral española», Economía Industrial, 333, 101-116.

Valdaliso, J. M. (2003) «Crisis y reconversión de la industria de construcción naval en el País Vasco», Ekonomiaz: Revista Vasca de Economía, 54, 52-67. 
Valdaliso, J. M. (2010) «Innovación y cooperación: la internacionalización de las empresas del "cluster" de la electrónica y las TICs en el País Vasco (c. 1970-2007)», Revista de Historia Industrial, 43, 163-191.

Valdaliso, J. M. (2013) «Las estrategias de desarrollo económico del País Vasco una perspectiva histórica», Ekonomiaz: Revista Vasca de Economía, 83, 146-174.
Vázquez, J. A. (2004) «El caso de Ensidesa», en González González, M. J. (ed.). Hierro y acero ante la mundialización: una perspectiva histórica. Avilés: Aceralia, pp. 161-179.

Velasco, R. y Plaza, B. (2003) «La industria española en democracia, 1978-2003», Economía industrial, 349-350, 155-180.

\section{Anexo 1}

Peso de los subsectores sobre el conjunto metalmecánico asturiano, 1978 y 2000

\begin{tabular}{lccc}
\hline & \multicolumn{3}{c}{$\mathbf{1 9 7 8}$} \\
\hline & Empleo & Producción & VAB \\
\hline Fabricación de productos metálicos & $50 \%$ & $51 \%$ & $51 \%$ \\
\hline Maquinaria, equipo, electrónica y precisión & $26 \%$ & $25 \%$ & $26 \%$ \\
\hline Material de transporte & $24 \%$ & $24 \%$ & $23 \%$ \\
\hline
\end{tabular}

\begin{tabular}{ccc}
\hline & $\mathbf{2 0 0 0}$ \\
\hline Empleo & Producción & VAB \\
\hline $50 \%$ & $47 \%$ & $50 \%$ \\
\hline $36 \%$ & $31 \%$ & $34 \%$ \\
\hline $14 \%$ & $22 \%$ & $16 \%$ \\
\hline
\end{tabular}

Fuente: elaboración propia con datos de SADEI, Tablas Input-Output.

\section{Anexo 2}

Empresas metalmecánicas en Asturias con más de 150 empleados, 1980 y 2000

\begin{tabular}{lcc}
\hline \multicolumn{1}{c}{ NOMBRE } & $\mathbf{1 9 8 0}$ & \\
\hline \multicolumn{1}{l}{ CNAE-74 } & PLANTILLA \\
\hline S. M. DURO FELGUERA (DIQUE) & 371 & 2.343 \\
\hline FÁBRICA NACIONAL DE CAÑONES Y FÁBRICA DE ARMAS & 329 & 2.184 \\
\hline S.A. JULIANA & 371 & 1.259 \\
\hline EMPRESA N. ALUMINIO S.A. & 224 & 984 \\
\hline SDAD.IND.ASTURIANA STA.BÁRBARA & 316 & 847 \\
\hline ELÉCTRICO I. CRADY S.A. & 342 & 686 \\
\hline ASTILLEROS DEL CANTÁBRICO & 371 & 564 \\
\hline AVELLO S.A. & 383 & 400 \\
\hline TALLERES DE MOREDA S.A. & 325 & 288 \\
\hline FUNDICIÓN NODULAR S.A. & 325 & 241 \\
\hline ARMSTRONG AMORTIGUADORES & 363 & 221 \\
\hline M. TUBERÍA INDUSTRAL, S.L. & 312 & 190 \\
\hline INDUSTRIAL AVILÉS S.A. & 311 & 186 \\
\hline FUENTE TRUBIA S.A. & 311 & 170 \\
\hline AGUINACO S.A. & 311 & 164 \\
\hline
\end{tabular}

Fuente: SADEI a través de FEMETAL, con datos de la Tesorería de la Seguridad Social.

\begin{tabular}{lcc}
\hline \multicolumn{1}{c}{ NOMBRE } & $\mathbf{2 0 0 0}$ & \\
\hline GRUPO DURO FELGUERA SA & CNAE-93 & PLANTILLA \\
\hline EMPRESA NAL. SANTA BÁRBARA INDUSTRIAS MI & 2951 & 1.571 \\
\hline HIERROS Y APLANACIONES SA & 2960 & 793 \\
\hline ESMENA SA & 2811 & 286 \\
\hline THYSSEN NORTE SA & 2811 & 255 \\
\hline METALÚRGICA ASTURIANA, S.A. & 2952 & 244 \\
\hline TREFILERIA MOREDA SA & 2821 & 233 \\
\hline FUNDICIÓN NODULAR, S.A. & 2873 & 228 \\
\hline MEFASA (MECAN. Y FABRIC. S.A.) & 2951 & 220 \\
\hline SAMOA INDUSTRIAL SA & 2956 & 188 \\
\hline
\end{tabular}

Fuente: SADEI a través de FEMETAL, con datos de la Tesorería de la Seguridad Social. 